\title{
EDITORIAL
}

\section{Evaluation and application of alternative air pollution exposure metrics in air pollution epidemiology studies}

\author{
Journal of Exposure Science and Environmental Epidemiology (2013) \\ 23, 565; doi:10.1038/jes.2013.50
}

Periodic review, revision and subsequent implementation of the National Ambient Air Quality Standards for criteria air pollutants rely upon various types of scientific air quality, exposure, toxicological dose-response and epidemiological information. Exposure assessment has a pivotal role in the evaluation of concentration-effects relationships for air pollutants during both standard setting process and in the selection of optimal risk mitigation strategies. In the past, epidemiological studies of air pollution have traditionally relied upon surrogates of personal exposures, such as ambient concentration measurements from fixed-site monitors. However, these central site-based exposure surrogates may not adequately capture the spatial and temporal variability of ambient pollutant concentrations, or account for exposures in different microenvironments (for example, indoors and in-vehicle), where pollutant infiltration and proximity to local sources can substantially have an impact on total exposures to ambient pollution. The potential exposure misclassification resulting from using central site measurements may also influence the strength and significance of the inferences derived from ambient pollution epidemiological studies. The 11 research articles published in this issue of JESEE compare the performance of a variety of alternative exposure metrics developed for particulate matter and gaseous pollutants, in the context of their applications to existing epidemiological health databases. The majority of the research reported in this issue involved collaborations between the scientists from EPA and various academic, federal and state institutions (including Emory University, Rutgers University, Rochester University, North Carolina State University, Lawrence Berkeley National Laboratory, King's College at London, New York State Department of Health, NIH and University of Florida).

Alternative exposure metrics were derived using a wide variety of approaches: central site or interpolated monitoring data; regional pollution levels based on measurements or air quality models; local scale air quality models; hybrid approaches that combine models; statistically blended modeling (that is, centralsite monitoring data with satellite-based aerosol optical depth information); ambient concentrations adjusted by home infiltration rates; and population-based human exposure models. Health outcomes considered include daily respiratory hospital admissions in the New York City, daily hospital emergency department visits in Atlanta, daily myocardial infarctions across the state of New Jersey and acute exacerbation of COPD in Cleveland. The various metrics were compared in their ability to characterize the spatial and temporal variation in exposure to multiple ambient air pollutants across different study areas. These metrics were then used to compare and contrast the estimated associations between short-term average ambient air pollution and acute morbidity or mortality outcomes.

Overall, results show that when compared with the use of central-site monitoring data, the enhanced spatial resolution in air quality or exposure predictions can sometimes influence pollutant-specific health effect estimates. The utility of these enhanced exposure assessment approaches depends on the influence of infiltration and human activity patterns on the pollutant concentrations, the spatial and temporal patterns of the pollutants of interest, and the form of the epidemiological study design chosen (for example, time-series or cross-sectional designs). For future individual or multipollutant air pollution exposure and health effects investigations, researchers recommend the development of pollutant-specific infiltration data (including for PM species), improved characterization of human time-activity patterns and refined prediction methods for exposures to local sources. We also encourage the broader use of alternative exposure metrics in future epidemiological studies of both shortand long-term effects of air pollution. However, we recognize that taking such a systematic approach to producing enhanced exposure estimates for health effects studies can be challenging. Nevertheless, to produce more reliable or relevant results for research and regulatory use will require a strong and sustained collaboration between the exposure scientists and epidemiologists, starting from the planning phase. With recent advances in the field of both exposure sciences and epidemiological analysis, we see great opportunities ahead for building on these successful multidisciplinary interactions.

\section{CONFLICT OF INTEREST}

The authors declare no conflict of interest.

\section{ACKNOWLEDGEMENTS}

We are grateful to Dr. Kathie Dionisio (EPA/NERL) for her important logistical support to the guest editors and to the authors. We also thank the researchers who contributed to this special issue and to the JESEE Editorial Board for their support and guidance during the production of this issue. Finally, we are grateful to Dr. Morton Lippmann, the Editor-In-Chief of JESEE, for encouraging us to pursue this important topic, dealing with evaluation and application of alternative air pollution exposure metrics in air pollution epidemiology studies.

\section{DISCLAIMER}

Although this work was reviewed by EPA and approved for publications, it may not necessarily reflect official Agency policy.

Halûk Özkaynak ${ }^{1}$, Lisa K. Baxter ${ }^{1}$ and Janet Burke ${ }^{1}$

${ }^{1}$ U.S. EPA, National Exposure Research Laboratory, (E205-01), Research Triangle Park, North Carolina, USA Email: ozkaynak.haluk@epa.gov 